\title{
ADHESIVE CAPSULITIS; MANAGEMENT BY PHYSIOTHERAPY VERSUS INTRA-ARTICULAR CORTICOSTEROID INJECTION
}

\author{
Muhammad Umair Hashmi, Babar Bakht Chughtai, Muhammad Nadeem Ahsan \\ Bahawal Victoria Hospital, Bahawalpur Pakistan
}

\begin{abstract}
Objective: To determine outcomes of intra-articular corticosteroids injection versus physiotherapy for the treatment of adhesive capsulitis using mean pain score on the visual analogue scale.

Study Design: Comparative prospective study.

Place and Duration of Study: Orthopedic outpatient department, Bahawal Victoria Hospital Bahawalpur from Jan to Jun 2021. Methodology: A total of 120 cases having adhesive capsulitis (frozen shoulder) were included in the study according to inclusion criteria. Non-probability consecutive sampling technique was used for the selection of cases. Patients were divided into two groups, group-A and group B, each containing 60 cases. Patients in group-A were given intra-articular steroid injection ( $2 \mathrm{ml}$ triamcinolone $40 \mathrm{mg} \pm 2 \mathrm{ml}$ of bupivacaine). Patients in group B received ten sessions of physiotherapy by a welltrained physiotherapist under the supervision of an orthopaedic surgeon on alternate days. After six weeks, outcomes were measured in terms of pain score using a visual analogue pain scale.

Results: Significant improvement was seen among patients in group-A with mean pain score from $7.32 \pm 0.89$ measured initially to $5.44 \pm 1.37$ measured after six weeks $(p<0.001)$. No significant improvement was found among patients in group B with a mean pain score of $7.58 \pm 0.94$ measured initially to $7.12 \pm 0.88$ measured after six weeks $(p>0.05)$.

Conclusion: Significant improvement in pain relief can be achieved using intra-articular steroid injection administered in the shoulder as compared to supervised sessions of physiotherapy among patients with adhesive capsulitis.
\end{abstract}

Keywords: Adhesive capsulitis, Frozen shoulder, Intraarticular injection, Shoulder physiotherapy, Visual analogue pain scale.

How to Cite This Article: Hashmi MU, Chughtai BB, Ahsan MN. Adhesive Capsulitis; Management by Physiotherapy Versus Intra-Articular Corticosteroid Injection. Pak Armed Forces Med J 2021; 71(5): 1824-1827. doi: https://doi.org/10.51253/pafmj.v71i5.5974

\footnotetext{
This is an Open Access article distributed under the terms of the Creative Commons Attribution License (https://creativecommons.org/licenses/by-nc/4.0/), which permits unrestricted use, distribution, and reproduction in any medium provided the original work is properly cited.
}

\section{INTRODUCTION}

Adhesive capsulitis is a painful condition in which shoulder movement becomes limited. In this condition, the joint capsule becomes inflamed, stiff and thick. It is associated with spontaneous onset of pain, restricted movements due to contracture of the capsule. It is a very common problem with an incidence of $2 \%$ reported in the general population and $30 \%$ reported in patients with diabetes mellitus ${ }^{1}$. Adhesive capsulitis (AC) can be primary also called idiopathic without any reported aetiology or factor triggering a chronic inflammatory response and increased fibroblast proliferation forming excessive scar tissue or adhesions in glenohumeral joint ${ }^{2}$. It is mostly found in obese and overweight people with age usually $<50$ years ${ }^{3}$. Secondary adhesive capsulitis is caused due to diabetes mellitus, shoulder injury, cerebrovascular accidents, cardiovascular disease or rotator cuff injury. It is rare among children, more common among males than the female population. Its prevalence is $2-5 \%$ among people with age $40-60$ years ${ }^{4}$. Limited active and

Correspondence: Dr Muhammad Umair Hashmi, Department of Orthopedic, Bahawal Victoria Hospital, Bahawalpur Pakistan

Received: 01 Jan 2021; revision received: 26 Jun 2021; accepted: 23 Jul 2021 passive range of motion of shoulder with pain make the diagnosis of $\mathrm{AC}$.

It is a self-limiting disease comprising of three stages. In the first stage, the patient has painful movements, in the second stage, movements are decreased and the last third stage is the recovery stage. Their symptoms last for an average period of 30 months ${ }^{5}$. Painful movements of the shoulder joint disturb normal activities of routine life. There are different treatment options for this condition according to various studies. Initially, NSAIDs and acetaminophen can be used for pain relief ${ }^{6}$. Other options include physical therapy, intraarticular corticosteroid injection, manipulation of shoulder joint under anaesthesia and arthroscopic release of the joint capsule, while physiotherapy helps in achieving a pain-free range of motion and it is a much effective treatment option ${ }^{7}$. Few studies report no satisfactory outcome by physical therapy alone and reported that intra-articular corticosteroids injection gives better outcomes in terms of reduced pain and improved range of motion as compared to physiotherapy ${ }^{8,9}$. Ultrasound-guided hydrodistention of joint followed by serial sessions of physiotherapy has been proved much effective ${ }^{10}$. Different studies recommend 
different treatment options, hence still it is unclear that which option is better ${ }^{8-10}$. According to our knowledge, limited studies have been done in Pakistan regarding the treatment of adhesive capsulitis using intraarticular corticosteroids injection compared to physiotherapy in terms of mean pain score. We hypothesize that intraarticular corticosteroids injection is more effective and provide a quick improvement in adhesive capsulitis than physiotherapy alone.

\section{METHODOLOGY}

After taking approval from institutional ethical review committee (ref. No 448, dated: 30-11-2020) 120 patients with adhesive capsulitis (in freezing phase of AC) were selected for study using no-probability consecutive sampling technique. This was a comparative prospective study. Patients presenting to orthopedic outpatient door Bahawal Victoria Hospital Bahawalpur from January to June 2021 were included in the study as per inclusion criteria. Sample size was calculated using WHO sample size formula Z2PQ/D2 (where $p=20 \%)^{4}$.

Inclusion Criteria: Patients of either gender, having age 18-70 years with shoulder pain without any known reason from more than three weeks but less than six months were included in the study after making diagnosis on the bases of history points like spontaneous onset of shoulder pain and limited passive range of motion in at least two planes.

Exclusion Criteria: Patients having previous history of any sort of surgery around the shoulder, history of trauma to shoulder, history of intra-articular steroids injection previously, having degenerative joint disease, osteoporosis, neuromuscular disorders, radiculopathy or referred pain from prolapsed intervertebral disc or already taking successful treatment for adhesive capsulitis, we-re excluded from the study.

Plain radiographs (antero-posterior view in neutral rotation, internal and external rotation, axillary lateral view and scapular-Y view) were taken to exclude any other shoulder pathology. Shoulder ultrasound and baseline investigations done like complete blood count, fasting and random blood sugar level, glycosylated hemoglobin $(\mathrm{HbA1c})$ level and thyroid profile to determine any possible cause of adhesive capsulitis. Informed written consent was taken from the patients in study group. Patients were divided into two groups group-A and group-B using lottery method, each containing 60 patients. Patients in group-A were given intra-articular Triamcinolone (corticosteroid) 40mg mixed with bupivacaine $2 \mathrm{ml}$ injection via posterior approach under aseptic measures by orthopedic consultant without using imaging technique. While eight sessions of physiotherapy on alternate days were given to the patients in group-B by a trained physiotherapist under supervision of orthopedic consultant. Each session was comprised on ultrasound therapy, TENS (Transcutaneous electrical nerve stimulation), range of motion (ROM) and continuous passive motion exercises. Patients of both groups were given same home exercises and same analgesics. At six-week follow-up all patients were evaluated for success of treatment by measuring mean pain score using visual analogue pain scale. In this study $57(47.5 \%)$ patients were laborers (maids and servants), 38 (31.6\%) were doing desk job and $25(20.8 \%)$ were housewives.

Data was collected on a self-designed proforma. Data was analyzed using statistical software SPSS-20. Descriptive statistical analysis was done for qualitative and quantitative variables. For quantitative variables like age and pain score, means and standard deviation were determined and for qualitative variables like gender, diabetes mellitus frequency and percentages were determined. Chi square test was applied on the qualitative data. Statistical significance was determined using independent t-test for quantitative data. The $p$ value of $\leq 0.05$ was considered statistically significant.

\section{RESULTS}

Total 120 cases included in this study were divided into two groups group-A and group-B, each containing 60 cases. Overall, male cases were $54(45 \%)$ and female cases were 66 (55\%) (Table-I). In group-A 26 $(43.3 \%)$ were male and $34(56.6 \%)$ were female cases, right shoulder was most commonly involved in 39 $(65 \%)$ cases. Mean age of the patients in group-A was $47.35 \pm 7.8$ years. In group-B, $28(46.6 \%)$ were male and $32(53.3 \%)$ were female cases, mean age of the patients was $46.21 \pm 6.4$ years and right shoulder involvement was more commonly found in $42(70 \%)$ cases (Table-I).

In group-A mean pain score was improved significantly from $7.43 \pm 0.98$ to $5.12 \pm 0.739$ after six

Table-I: Basic characteristics of patients in group-A and B.

\begin{tabular}{|c|c|c|c|}
\hline Characteristics & $\begin{array}{c}\text { Group-A } \\
(n=60)\end{array}$ & $\begin{array}{c}\text { Group-B } \\
(n=60)\end{array}$ & $\begin{array}{c}p- \\
\text { value }\end{array}$ \\
\hline \multicolumn{4}{|l|}{ Gender } \\
\hline Male & $26(43.3 \%)$ & $28(46.6 \%)$ & \multirow{2}{*}{0.687} \\
\hline Female & $34(56.6 \%)$ & $32(53.3 \%)$ & \\
\hline Mean age (years) & $47.35 \pm 7.8$ & $46.21 \pm 6.4$ & 0.176 \\
\hline \multicolumn{4}{|c|}{ Shoulder Side Involvement } \\
\hline Right & $39(65 \%)$ & $42(70 \%)$ & \multirow{3}{*}{0.590} \\
\hline Left & $17(28.3 \%)$ & $15(25 \%)$ & \\
\hline Both & $04(6.6 \%)$ & $03(5 \%)$ & \\
\hline
\end{tabular}


weeks of treatment with intra-articular corticosteroid injection, while no significant improvement was reported in group-B patients after ten sessions of physiotherapy with a mean pain score of $7.51 \pm 0.947$ initially to $7.02 \pm 1.066$ when assessed on six weeks follow-up (Table-II).

Table-II: Improvement in mean pain score in both groups measured on visual analogue scale.

\begin{tabular}{l|c|c|c}
\hline & $\begin{array}{c}\text { Group-A } \\
(\mathbf{n = 6 0 )}\end{array}$ & $\begin{array}{c}\text { Group-B } \\
(\mathbf{n = 6 0 )}\end{array}$ & $\begin{array}{c}\boldsymbol{p} \text { - } \\
\text { value }\end{array}$ \\
\hline Initial Visual Analogue & $7.43 \pm$ & $7.51 \pm$ & 0.656 \\
Score (Mean \pm SD) & 0.980 & 0.947 & \\
\hline Visual Analogue Score & $5.12 \pm$ & $7.02 \pm$ & $<0.001$ \\
after 6 weeks (Mean \pm SD) & 0.739 & 1.066 & \\
\hline
\end{tabular}

Most of the cases (46\%) were having age 41-60 years in our study group. This disease was not uncommon (23\%) among the patients with age 21-40 years. It was seen that this disease is more common in old age patients (Figure).

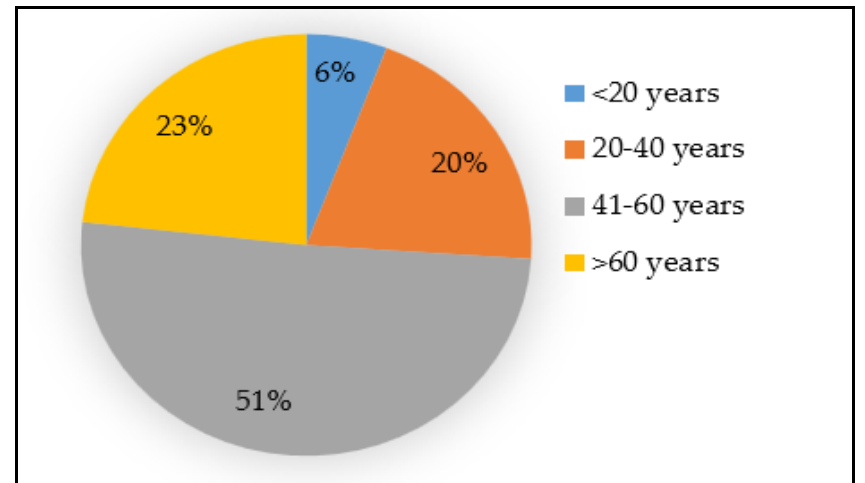

Figure: Age distribution of cases in study group (Group-A \& B) $(\mathrm{n}=120)$.

\section{DISCUSSION}

Adhesive capsulitis is a common cause of shoulder pain mainly in old age people. Conservative treatment in the form of physiotherapy and intraarticular corticosteroids injection is much effective in the majority of cases ${ }^{11}$. However, other measures like manipulation under anaesthesia and arthroscopic release of shoulder capsule can be done in those not responding to conservative treatment ${ }^{12,13}$. It is very common in people between $60-70$ years of age $33.3 \%{ }^{14}$. In our study majority of cases (51\%) were between 40 60 years of age and 23\% cases were between $60-70$ years of age. However, results are similar as most of the cases were old age. Many studies show adhesive capsulitis more common in the female gender ${ }^{15}$. Sattar et al found adhesive capsulitis more common in females $(60 \%)$ than males $(40 \%)$. He found a strong association of female gender ${ }^{16}$. In our study female cases were more (55\%) than male cases $(45 \%)$ with male to female ratio of 1:1.2. It usually involves the dominant side due to increased use of that side ${ }^{17}$. A study was conducted in Turkey on 64 patients in which $56.6 \%$ cases were having right shoulder and 50\% were having left shoulder adhesive capsulitis ${ }^{18}$. These results are similar to our findings where the right shoulder was involved in $67.5 \%$ of cases and the left shoulder was involved in $26.6 \%$ of cases. Studies conducted by physiotherapists have emphasized physiotherapy more than other modes of treatment and proved the role of deep heat and stretching exercises superior to invasive procedures in relieving pain and improving range of motion in $\mathrm{AC}^{19}$. A study conducted by Rawat et al. Reported significant improvement in $84.38 \%$ cases on six-week follow-up and improvement in all $100 \%$ cases on $12^{\text {th }}$-week follow-up measured on VAS Score after intra-articular corticosteroid injection ${ }^{20}$. In our study six weeks, follow-up was done after intra-articular corticosteroid injection and significant improvement was found in all $(100 \%)$ cases as measured on VAS score. Another study conducted in Taiwan by Lin et al reported improvement in pain and shoulder function after an intra-articular steroid injection and shoulder distension in the medium term (6-16 weeks) but no significant benefits in the long term. He concluded that early distension can be the treatment of choice when the main complaint of the patient is limited external rotation of the shoulder ${ }^{21}$. A national study conducted at the Orthopedic department of Benazir Bhutto Hospital Rawalpindi by Butt et al reported improvement in VAS score from $3.33 \pm 1.1$ to $2.25 \pm 0.78$ after intra-articular corticosteroid injection on $4^{\text {th }}$-week follow-up. He found intra-articular steroid injection treatment of choice and safer than manipulation under anaesthesia and physiotherapy in the freezing phase of adhesive capsulitis ${ }^{22}$. Aziz et al studied outcomes of intra-articular corticosteroid injection and physiotherapy at Combined Military Hospital Kohat, Pakistan and reported improvement in shoulder pain measured on a visual analogue pain scale from $7.2 \pm$ 0.91 to $5.57 \pm 1.23$ after intra-articular corticosteroid injection and no significant improvement was found after eight sessions of physiotherapy on $6^{\text {th }}$ week follow-up ${ }^{23}$. These results were similar to our findings where significant improvement was observed on VAS from $7.43 \pm 0.980$ to $5.12 \pm 0.739$ after intra-articular corticosteroid injection after six weeks follow-up. In this study, we administered intra-articular injection without imaging modalities but the confirmation of injection site can be done using ultrasound or fluoro- 
scopy. Our study is based on the best available evidence but its results cannot be generalized to any intraarticular injection or physiotherapy technique except what we have used in this study. Still, further studies are needed to be done in our set-ups to evaluate longterm outcomes of intra-articular corticosteroid injection and physiotherapy.

\section{CONCLUSION}

Intra-articular corticosteroid injection technique is a treatment of choice in adhesive capsulitis which provides significant improvement in pain relief as compared to supervised sessions of physiotherapy alone which show no significant relief in pain.

Conflict of Interest: None.

\section{Authors' Contribution}

MUH: Conception and data collection. BBC: Data analysis and interpretation. MNA: Data collection.

\section{REFERENCES}

1. Ranalletta M, Rossi LA, Bongiovanni SL, Tanoira I, Elizondo $\mathrm{CM}$, Maignon GD. Corticosteroid injections accelerate pain relief and recovery of function compared with oral NSAIDs in patients with adhesive capsulitis: a randomized controlled trial. Am J Sports Med 2016; 44(2): 474-481.

2. Le HV, Lee SJ, Nazarian A, Rodriguez EK. Adhesive capsulitis of the shoulder: a review of pathophysiology and current clinical treatments. J Shoulder Elb Surg 2017; 9(2): 75-84.

3. Kingston K, Curry EJ, Galvin JW, Li X. Shoulder adhesive capsulitis: epidemiology and predictors of surgery. J Shoulder Elb Surg 2018; 27(8): 1437-1443.

4. Asheghan M, Aghda AK, Hashemi E, Hollisaz M. Investigation of the effectiveness of acupuncture in the treatment of the frozen shoulder. Material socio-medica 2016; 28(4): 253-260.

5. Uppal HS, Evans JP. Frozen shoulder: A systematic review of therapeutic options. World J Orthop 2015; 6(2): 263-268.

6. RajKhare Y, Sah S, Rai RK, Subedi N, Adhikari AR. outcomes of short-course oral corticosteroid with nonsteroidal anti-inflammatory drugs for early-stage of the frozen shoulder at koshi zonal hospital. Med Res Chronicles 2018; 5(3): 246-251.

7. Brun SP. Idiopathic frozen shoulder. Aust J Gen Pract 2019; 48(11): 757-761.

8. Eljabu W, Klinger HM, von Knoch M. Prognostic factors and therapeutic options for treatment of frozen shoulder: a systematic review. Arch Orthop Trauma Surg 2016; 136(1): 1-7.

9. Cho $\mathrm{CH}$, Jin $\mathrm{HJ}$, Kim DH. Comparison of clinical outcomes between idiopathic frozen shoulder and diabetic frozen shoulder after a single ultrasound-guided intra-articular corticosteroid injection. Diagnostic 2020; 10(6): 370-376.
10. Bryant M, Gough A, Selfe J. The effectiveness of ultrasoundguided hydrodistension and physiotherapy in the treatment of frozen shoulder/adhesive capsulitis in primary care: a single centre service evaluation. J Shoulder Elb 2017; 9(4): 292-298.

11. Kraal T, Boer R, van den Borne MP, Koenraadt K, Goossens P, Eygendaal D. Manipulation under anaesthesia versus physiotherapy treatment in stage two of a frozen shoulder: a study protocol for a randomized controlled trial. BMC Musculoskelet Disord 2017; 18(1): 412-418.

12. Kanbe K. Clinical outcome of arthroscopic capsular release for frozen shoulder: essential technical points in 255 patients. J Orthop Surg Res 2018; 13(1): 56-60.

13. Hagiwara Y, Ando A, Kanazawa K, Koide M, Sekiguchi T, Hamada J, et al. Arthroscopic coracohumeral ligament release for patients with frozen shoulder. Arthrosc Tech 2018; 7(1): e1-e5.

14. Al-Hashimi RA. Analytical observational study of frozen shoulder among patients with diabetes mellitus. Joints 2018; 6(3): 141-48.

15. Chellathurai A, Subbiah K, Elangovan A, Kannappan S. Adhesive capsulitis: MRI correlation with clinical stages and proposal of MRI staging. Indian J Radiol Imaging 2019; 29(1): 19-24.

16. Sattar A, Shabbir M, Faisal Z, Ayaz M, Muhammad W. Outcome of intra-articular corticosteroid in the treatment of chronic adhesive capsulitis. Pak J Surg 2019; 35(4): 331-334.

17. Manske RC, Prohaska D. Diagnosis and management of adhesive capsulitis. Curr Rev Musculoskelet Med 2008; 1(3-4): 180-189.

18. Alptekin HK, Aydın T, İflazoğlu ES, Alkan M. Evaluatıng the effectiveness of frozen shoulder treatment on the right and left sides. J Phys Ther Sci 2016; 28(1): 207-122.

19. Mertens MG, Struyf F, Meert L, Lauwers M, Schwank A, Verborgt $\mathrm{O}$, et al. Factors influencing treatment outcome of physical therapy in frozen shoulder patients: a systematic review. J Back Musculoskelet Rehabil 2021; 34(2): 195-205.

20. Rawat MS, Juyal A, Agarwal A. Evaluation of the role of intraarticular steroid injection in frozen shoulder. Int J Orthop Sci 2018; 4(1): 792-794.

21. Lin MT, Hsiao MY, Tu YK, Wang TG. Comparative efficacy of intra-articular steroid injection and distension in patients with frozen shoulder: a systematic review and network meta-analysis. Arch Phys Med Rehab 2018; 99(7): 1383-1394.

22. Butt MI, Iqbal T, Anjum S. Comparison between manipulation under anaesthesia and intra-articular steroid injections for frozen shoulder. J Rawal Med Col 2018; 22(4): 342-345.

23. Aziz T, Khan AA, Akhtar N, Ayyub A, Iqbal S, Shafaat HK. intra-Articular corticosteroids versus physiotherapy in the management of adhesive capsulitis. Pak Armed Forces Med J 2018; 68(3): 565-569.

24. Inayat F, Ali NS, Shahid H, Younus F. Prevalence and determinants of frozen shoulder in patients with diabetes: a single-centre experience from Pakistan. Cureus 2017; 9(8): e1544. 